\title{
La mortalidad por cáncer de próstata en andalucía: aportaciones al cribado poblacional
}

\author{
M. Ruiz-Ramos*, A. Escolar Pujolar** \\ *Registro de Mortalidad de Andalucía. **Servicio Medicina Preventiva y Salud Pública. \\ Hospital Universitario "Puerta del Puerta del Mar". Cádiz.
}

Actas Urol Esp 2005; 29 (1): 41-46

\section{RESUMEN}

LA MORTALIDAD POR CÁNCER DE PRÓSTATA EN ANDALUCÍA: APORTACIONES AL CRIBADO POBLACIONAL Fundamento: Describir la mortalidad por cáncer de próstata en Andalucía desde 1975 al 2001.

Métodos: Se han calculado tasas brutas, tasas ajustadas por edad, Años Potenciales de Vida Perdidos (APVP), el riesgo de morir por cáncer de próstata y estimado los cambios producidos en las tasas ajustadas.

Resultados: El número de muertes por cáncer de próstata aumentó de 407 a 767 . Las tasas brutas pasaron de 13,54 a 21,35 por 100.000 personas. Sin embargo, las tasas ajustadas por edad mostraron unos valores relativamente estables a lo largo de la serie, con un porcentaje anual de cambio de $-0,21 \%$ y los indicadores de mortalidad prematura descendieron. El riesgo acumulado de morir por cáncer de próstata aumentó con la edad, experimentando a partir de los 50 años un incremento exponencial, tanto en el periodo de 1975-1979 como en el 1997-2001, con valores superiores en el segundo periodo respecto al primero.

Conclusiones: El cáncer de próstata en Andalucía ha aumentado en valores absolutos debido al envejecimiento de la población. Al controlar la edad la mortalidad ha permanecido estable, con un desplazamiento de las muertes hacia los grupos más extremos de la vida, como señalan la disminución de las muertes prematuras. Los resultados de este trabajo no apoyan la puesta en marcha de programas de cribado poblacional.

Palablas clave: Cáncer de próstata. Mortalidad. Cribado poblacional. Antígeno prostático específico.

\section{ABSTRACT}

THE MORTALITY OF PROSTATE CANCER IN ANDALUSIA. CONTRIBUTIONS TO MASS SCREENING.

Background: To describe the mortality of prostate cancer in Andalusia from 1975 to 2001.

Methods: Estimation of crude rates, age-adjusted rates, Potential Years of Life Lost (PYLL) adjusted rates, risk of death and the changes produced in the adjusted rates.

Results: Prostate cancer deaths rise from 407 to 767 . Crude rates increased from 13,54 to 21,35 per 100.000 persons-years. However, the age-adjusted rates showed a steady trend over the study period, with an annual percent change of $-0,21 \%$, and the premature mortality indicators declined. The cumulative risk of death increased with age, and presented an exponential increase after the age of 50 years, in both the periods, 1975-1979 as 1997-2001, with highest values in the second one.

Conclusions: Prostate cancer mortality in Andalusia has increased in absolute values due to the ageing of the population. When adjusted for age, the mortality has remained stable, with a shift of deaths toward the more extreme age groups, as indicated by the decrease of premature deaths. The results of this study do not support the starting-up of mass screening programs.

Key words: Prostate cancer. Mortality. Mass screening. Prostate-specific antigen.

$\mathrm{E}_{\mathrm{p} s \mathrm{sen}}^{1}$ cáncer de próstata (CP) ocupa la segunda posición como causa de muerte por cáncer en los hombres de algunos países desarrollados. En Estados Unidos se estimó que 189.000 hombres fueron diagnosticados de esta enfermedad en el año 2002, de los cuales 30.200 murieron ${ }^{1}$. La mortalidad varia ampliamente ${ }^{2}$, teniendo las tasas más elevadas los países caribeños, escandinavos, Bélgica y Holanda, y las más bajas China, Japón y los países de la antigua Unión Soviética.

En 1985, en la Unión Europea, se produjeron 43.177 muertes por este cáncer, esperándose para el 2000 una cifra de 55.800 muertes. Sin embargo en este último año se ha estimado un total de 60.000 muertes, es decir unas 4.200 más de las esperadas. Entre 1985 y el 2000 el riesgo de muerte por este tumor ha crecido un $5 \%$, crecimiento producido en todos los países excepto Francia, Italia, Luxemburgo y Alemania ${ }^{3}$. España junto con Grecia e Italia tienen la incidencia y mortalidad más baja de la Unión Europea. En nuestro país el número de casos de $\mathrm{CP}$ de diagnóstico en los últimos 5 años sería de 36.492 pacientes, de acuerdo a las estimaciones de la 
prevalencia realizadas por la Agencia Internacional de Investigación del Cáncer de la OMS (IARC-OMS) ${ }^{4}$.

En España el CP ocupa la tercera posición en la mortalidad, tras el cáncer de pulmón y el colorrectal, con 5.568 fallecimientos y una tasa ajustada por edad de 22,94 por 100.000 habitantes en 1999. También ocupa el tercer lugar en incidencia, con tasas estimadas para 1997 de 46,28 casos por 100.000 habitantes. La evolución de las tasas de mortalidad ha sido ascendente desde los años 50, si bien la tendencia se ha suavizado a partir de 1970. Globalmente se ha observado en el periodo 1952-1996 un incremento medio en torno al $1 \%$ anual, con un cambio en la tendencia en el año 1998, a partir del cual la mortalidad comienza a disminuir ${ }^{5}$.

El CP es una enfermedad muy asociada con el envejecimiento. No se diagnostica habitualmente en hombres menores de 50 años. A partir de esta edad la incidencia y la mortalidad se incrementan exponencialmente ${ }^{6}$. El riesgo de desarrollar CP es del doble para los que tienen a su padre o hermano afectados por esta patología, aumenta cuando hay más de un familiar de primer grado con $\mathrm{CP}$ y con presentación a edades tempranas ${ }^{7}$. $\mathrm{El}$ alto consumo de grasas, y carnes puede estar relacionado con la mayor incidencia en los países occidentales $^{8}$. Por el contrario el tomate y sus productos, el selenium contenido en los cereales, pescado y carne, así como los bajos niveles de insulina se han relacionado con baja incidencia ${ }^{9-11}$. No hay estudios que establezcan cuales son las causas del CP, y los factores de riesgo conocidos, edad y antecedentes familiares, no son modificables, con lo que las posibles estrategias de prevención quedarían centradas fundamentalmente en el diagnóstico precoz mediante programas de cribado poblacional, que sin embargo son considerados como de efectividad controvertida. Las estrategias de prevención relacionadas con cambios en los patrones de alimentación, aunque de efectividad cuestionable, podrian aportar beneficios importantes en otros ámbitos como el de las enfermedades cardiovasculares.

El objetivo de este trabajo es describir la situación actual y la tendencia de la mortalidad por cáncer de próstata en Andalucía desde 1975 al 2001, para que sirva para orientar las decisiones preventivas que se puedan tomar, y específicamente las relacionadas con la posible puesta en marcha de programas poblacionales de detección precoz.

\section{PACIENTES Y MÉTODOS}

Los datos de mortalidad proceden de los Boletines Estadísticos de Defunción (BED) procesados por el Instituto Nacional de Estadística (INE) para los años de 1975 a $1991^{12}$, y por el Instituto de Estadística de Andalucía (IEA) ${ }^{13}$ para el periodo de 1992 a 2001. Se han incluido los códigos 185 de la Clasificación Internacional de Enfermedades (CIE) $8^{\mathrm{a}}$ y $9^{\mathrm{a}}$ revisión, que corresponden al tumor maligno de próstata. Para el periodo de 1999 a 2001 la revisión que se ha utilizado de la CIE ha sido la $10^{\mathrm{a}}$, que clasifica el CP con el código C61.

Las poblaciones necesarias para el cálculo de indicadores corresponden a las proyecciones realizadas por el INE, agrupadas en grupos quinquenales, excepto para los menores de 1 año, los de 1 a 4 años y los mayores de 85 años y más.

Los indicadores calculados han sido: las tasas brutas de mortalidad anuales por 100.000 personas-año; las tasas ajustadas por edad por 100.000 personas-año mediante el método directo de ajuste, utilizando como población de referencia la Población Estándar Europea; las tasas truncadas de 35 a 64 años ajustadas por edad. Este último tipo de tasa sólo tiene en cuenta los grupos de edad de 35 a 64 años, eliminando así la falta de precisión en los diagnósticos de cáncer sobre todo en las personas mayores. Además la probabilidad de morir a edades menores de 35 años es muy baja y puede distorsionar los resultados.

También se ha calculado el riego acumulado de morir por CP según el método de la tabla de vida $^{14,15}$ para 1975-1979 y 1997-2001: a partir de una cohorte ficticia se calcula, de forma sucesiva, para cada grupo de edad, el número de muertes por $\mathrm{CP}$ y las personas a riesgo en el siguiente grupo de edad son las que quedan vivas después de restar todas las muertes, las causadas por el cáncer y por otras causas. La probabilidad acumulada de morir se ha calculado sumando las personas que mueren por $\mathrm{CP}$ hasta un grupo de edad determinado y dividiendo por el 
número de personas que estaban a riesgo. Para calcular el riesgo acumulado durante toda la vida se supone que el último intervalo de edad, que es abierto, sigue una distribución exponencial y se estima el número de muertes que ocurrirán en este intervalo.

Por último se han calculado tasas ajustadas por edad mediante el método directo de los Años Potenciales de Vida Perdidos ${ }^{16}$ (APVP) entre $1 \mathrm{y}$ 70 años, como un indicador de mortalidad prematura. Cada uno de estos indicadores se ha obtenido para cada uno de los años de la serie.

El porcentaje anual de cambio se ha estimado mediante las tasas estandarizadas con la población europea. Para ello con las tasas del primer quinquenio (1975-1979) y las del último (19972001), se han calculado los logaritmos neperianos de las tasas, se ha restado al valor del último quinquenio el del primero y la diferencia se ha dividido por los 27 años del periodo estudiado y se ha multiplicado por cien.

\section{RESULTADOS}

La mortalidad por cáncer de próstata en Andalucía durante el periodo estudiado ha experimentado un aumento en números absolutos (Tabla 1), pasando de 407 a 767 defunciones entre 1975 y 2001 . Las tasas brutas de mortalidad también han sufrido un importante aumento, de 13,54 a 21,35 por 100.000 personas-año. Las tasas ajustadas por edad presentan un comportamiento distinto a los anteriores indicadores, habiendo descendido ligeramente, de 22,71 a 21,54 defunciones por 100.000 personas-año entre los años extremos del periodo estudiado. Los indicadores de mortalidad prematura también han descendido. Así, los APVP han pasado de una tasa de 32,05 en 1975 a 26,35 años por 100.000 personas-año en 2001, y las tasas truncadas entre 35 y 64 años de edad, de 5,03 a 4,92 por 100.000 personas-año.

En la Figura 1 se presentan las tasas de mortalidad ajustadas por edad. La evolución ha sido estable en el conjunto del periodo, observándose un pico de máxima mortalidad en el año 1982 con una tasa de 25,02 defunciones por 100.000 personas-año, que descendió hasta los valores más bajos en el año 1989 con una tasa de 19,53, produciéndose a partir de esta fecha un ascenso con fluctuaciones anuales.
Tabla 1

Indicadores de mortalidad por cáncer de próstata. Andalucía, 1975 a 2001

\begin{tabular}{|c|c|c|c|c|c|}
\hline Año & Casos $=$ & TB * & TED“ & $\mathbf{T T}^{\wedge}$ & APVP \\
\hline 1975 & 407 & 13,54 & 22,71 & 5,03 & 32,05 \\
\hline 1976 & 415 & 13,72 & 23,10 & 4,67 & 27,02 \\
\hline 1977 & 421 & 13,82 & 22,77 & 6,35 & 36,31 \\
\hline 1978 & 440 & 14,32 & 22,47 & 5,18 & 30,72 \\
\hline 1979 & 454 & 14,64 & 23,73 & 4,70 & 28,26 \\
\hline 1980 & 458 & 14,62 & 22,22 & 5,04 & 28,54 \\
\hline 1981 & 486 & 15,35 & 23,03 & 6,05 & 33,77 \\
\hline 1982 & 518 & 16,18 & 25,02 & 4,70 & 26,85 \\
\hline 1983 & 522 & 16,09 & 23,55 & 4,96 & 28,86 \\
\hline 1984 & 545 & 16,57 & 23,21 & 6,21 & 37,80 \\
\hline 1985 & 516 & 15,51 & 21,59 & 5,17 & 29,58 \\
\hline 1986 & 524 & 15,62 & 20,86 & 4,81 & 26,87 \\
\hline 1987 & 552 & 16,35 & 21,90 & 4,42 & 27,41 \\
\hline 1988 & 522 & 15,41 & 20,16 & 3,75 & 23,67 \\
\hline 1989 & 530 & 15,62 & 19,53 & 5,61 & 30,81 \\
\hline 1990 & 572 & 16,82 & 21,10 & 4,56 & 30,34 \\
\hline 1991 & 557 & 16,30 & 19,98 & 3,96 & 23,30 \\
\hline 1992 & 583 & 16,94 & 20,45 & 3,72 & 24,17 \\
\hline 1993 & 620 & 17,85 & 21,02 & 5,00 & 27,95 \\
\hline 1994 & 661 & 18,83 & 22,18 & 4,68 & 28,02 \\
\hline 1995 & 652 & 18,42 & 20,67 & 4,47 & 25,23 \\
\hline 1996 & 758 & 21,30 & 23,96 & 4,88 & 28,84 \\
\hline 1997 & 683 & 19,21 & 21,25 & 5,09 & 28,68 \\
\hline 1998 & 744 & 20,91 & 23,02 & 4,34 & 25,23 \\
\hline 1999 & 728 & 20,39 & 21,54 & 4,92 & 28,59 \\
\hline 2000 & 726 & 20,27 & 21,15 & 3,75 & 22,36 \\
\hline 2001 & 767 & 21,35 & 21,54 & 4,92 & 26,35 \\
\hline
\end{tabular}

= Casos: Número de defunciones.

* TB: Tasa bruta.

- TED: Tasa ajustada por edad a la población estandar europea.

^ TT: Tasa truncada ajustada por edad de 35 a 64 años.

APVP: Tasa ajustada por edad de Años Potenciales de Vida Perdidos entre 1 y 70 años.

En la Figura 2 se representa el riesgo acumulado de morir por cáncer de próstata en Andalucía en los periodos de 1975 a 1979 y de 1997 al 2001 por grupos de edad. Como puede observarse existe un aumento exponencial a partir de los 50-54 años, tanto en el primero como en el segundo periodo. A esta edad 1 de cada 5.802 andaluces murió por CP en el periodo de 1975 a 1979, aumentando ligeramente a 1 de cada 5374 en el último quinquenio, lo que supone una probabilidad del 0,02\% en ambos periodos. En el grupo de 85 y más esta probabilidad aumentó desde un 2,03\% en el primer quinquenio a un $2,55 \%$ en el segundo, lo que supuso 1 muerto por CP por cada 49 y 39 hombres respectivamente para el primer y último quinquenio. Comparando las dos curvas, en todos los grupos de edad se observa un mayor riesgo acumulado de morir en 1997-2001 que en el periodo 1975-1979. 


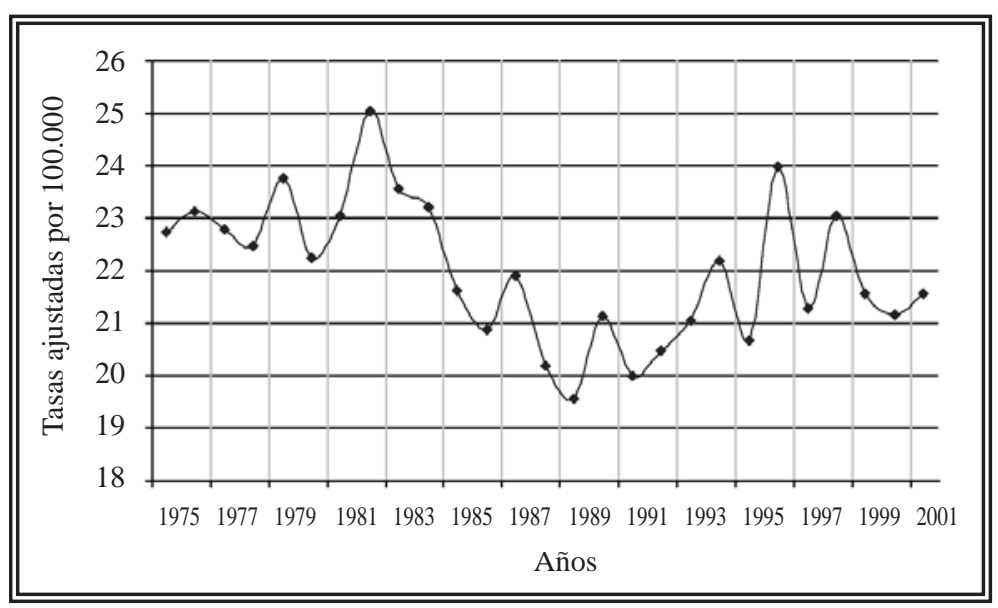

FIGURA I. Evolución de la mortalidad por cáncer de próstata en Andalucía entre 1975 y 2001. Tasas ajustadas por 100.000 personasaño, ajustadas por edad.

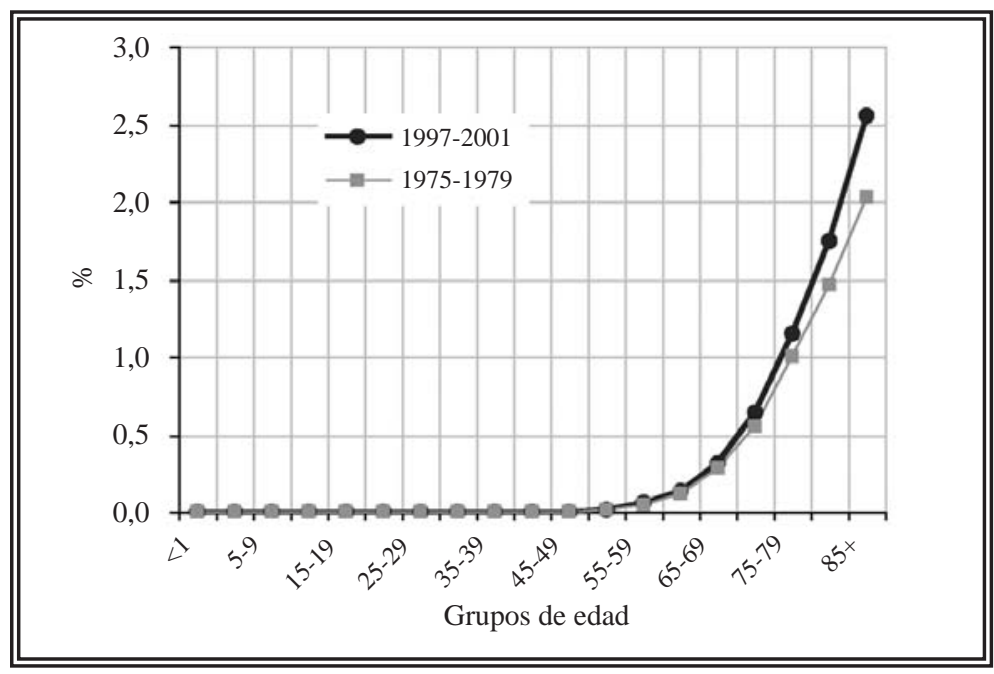

FIGURA II. Riesgos acumulados (en \%) de morir por cáncer de próstata en Andalucía, según periodo de defunción: 1975-1979 y 1997-2001.

de edad. Llama la atención la disparidad entre los indicadores brutos y los ajustados por edad. Los primeros (número de defunciones y tasa bruta) han sufrido un aumento considerable, que se justifica por el envejecimiento de la población andaluza a lo largo de los veintisiete años estudiados, que cambia de signo cuando se controla la edad a través del ajuste de tasas mediante el método directo. Estos indicadores ajustados, permiten hacer comparaciones entre diferentes periodos de tiempo y entre diferentes países o regiones, siempre que se utilice la misma población estándar para realizar los cálculos. En este trabajo se ha utilizado la población estándar europea, que tiene una estructura muy parecida a la actual de Andalucía, por eso las tasas brutas y ajustadas van teniendo un valor parecido en los últimos años de la serie. Sin embargo son unos indicadores que no representan el valor real de la mortalidad por CP en Andalucía, pues para ello se necesita conocer el número de defunciones y las tasas brutas.

Los indicadores de mortalidad prematura, tasas truncadas y tasas de APVP, se han mantenido en valores más estables que los citados en el párrafo anterior, aunque las tasas ajustadas de APVP ha descendido de una manera clara, lo que se debe interpretar como un desplazamiento de las muertes a los

El porcentaje promedio anual de cambio de las tasas de mortalidad ajustadas por edad de Andalucía durante los 27 años analizados, calculado con el primer y último quinquenio, ha descendido un $0,21 \%$ anual, aunque no es estadísticamente significativo.

\section{DISCUSIÓN}

La mortalidad por cáncer de próstata en Andalucía ha tenido una evolución desigual en el periodo estudiado, con una estabilización en los últimos años. Presenta una distribución por edad igual a la descrita en otros países y regiones, con un aumento exponencial a partir de los 50 años años extremos de la vida, posiblemente por un aumento de la supervivencia debido a un mejor tratamiento del CP.

Andalucía presenta unos niveles de mortalidad medios-bajos con respecto al conjunto de España ${ }^{17}$, con una tendencia estable, más clara en los últimos años estudiados, que podrían estar influidos por el "sesgo de atribución" y por la mejora en los tratamientos. El "sesgo de atribución" hace que algunas muertes en hombres con $\mathrm{CP}$ debidas a otras causas se atribuyan al CP. Si este porcentaje es estable la mortalidad podría estar reflejando aumentos o descensos en la incidencia de esta enfermedad en la población. 
Cambios en el tratamiento del $\mathrm{CP}$, como aumento de prostatectomías radicales, nuevos tratamientos hormonales, mejoras en la radioterapia, etc. podrían influir en una reducción en la mortalidad por $\mathrm{CP}^{18}$, pero habrá que esperar a los próximos años para comprobar si se confirma.

Otros resultados, aparentemente contradictorios, son el aumento del riesgo acumulado de morir por $\mathrm{CP}$ en todos los grupos de edad en el último quinquenio con respecto al primero, que estaría en contra de la evolución a la estabilización e incluso al ligero descenso como muestran los porcentajes anuales de descenso. Esto es debido a que este indicador mide las muertes por $\mathrm{CP}$ respecto al resto de causas de mortalidad, y está influido por un mayor descenso del riesgo acumulado de muerte del resto de causas en el segundo periodo que en el primero.

Desde el punto de vista de los programas de intervención sanitaria dirigidos a prevenir la aparición de nuevos casos (incidencia) de $\mathrm{CP}$ o al menos retrasar la muerte hasta los grupos más extremos de la vida, con la evidencia disponible, el planteamiento se centra de lleno entorno al cribado de CP, ya que es la actividad que en algunos lugares del mundo, con tasas de incidencia y mortalidad más elevadas que las de Andalucía y el conjunto de España, han demostrado cierta efectividad, aunque los resultados son contradictorios y discutibles. En Andalucía ${ }^{19}$ no se ha planteando por el momento esta posibilidad.

Uno de los problemas básicos para el cribado de este cáncer es su diversificación en tres entidades patológicas. La primera es una enfermedad latente que no produce daños para la salud y es un hallazgo casual muy frecuente en necropsias de personas de edad avanzada; la segunda es una enfermedad que progresará hasta dar síntomas y puede causar la muerte si no se trata; y la tercera muy agresiva, causará la muerte independientemente del tratamiento que se aplique. El cribado sólo será efectivo en la segunda forma de presentación patológica, que se estima representa entorno a un $8 \%$ de todos los casos y a través de la prueba de cribado no hay forma de diferenciar entre las tres formas de presentación ${ }^{20}$.

Se han propuesto tres pruebas de cribado: el tacto rectal, la detección de antígeno prostático específico (PSA) y la ecografía transrectal. La prueba más discutida actualmente es la del PSA. Esta prueba no es específica del cáncer, sino del tejido prostático, por lo que puede estar aumentada en cualquier proceso que altere el tamaño de la próstata. No están suficientemente establecidos los valores a partir de los cuales el resultado es positivo, ni su sensibilidad ni especifi$\operatorname{cidad}^{21,22}$. En cuanto a la reducción de la mortalidad, no hay suficientes evidencias que relacionen un descenso de la mortalidad con el cribado de $\mathrm{CP}^{23,24}$. El European Randomized Study of Screening for Prostate Cancer, puesto en marcha en $1994^{25}$, del que algunos grupos españoles han publicado resultados preliminares ${ }^{26} \mathrm{y}$ el Prostate, Lung, Colorectal and Ovarian Cancer Srcreening Trial (PLCO) en EEUU ${ }^{27}$, que completo en 2001 su fase de reclutamiento, suministrarán dentro de pocos años (2005-2008) información valiosa.

Por ahora, tanto el U.S. Preventive Task For$\mathrm{ce}^{28}$, como el recientemente revisado Código Europeo Contra el Cáncer ${ }^{29}$ concluyen que las actuales evidencias son insuficientes para la recomendación de la realización de la prueba del PSA como instrumento de detección precoz a nivel poblacional. Es importante distinguir entre los posibles beneficios individuales de una prueba de detección precoz, de los que puedan obtenerse para el conjunto de la población. Incluso si los resultados de los ensayos clínicos randomizados actualmente en marcha demostrasen reducciones en la mortalidad por cáncer de próstata, deberían ser considerados numerosos aspectos de tipo social, psicológicos, organizativos y económicos antes de la introducción de un programa poblacional de detección precoz basado en el PSA ${ }^{30,31}$. En la tabla 2 se resumen algunos de los argumentos en contra del uso del PSA como prueba de cribado poblacional.

De acuerdo con las evidencias respecto a la mortalidad y su evolución en Andalucía, no parece por ahora recomendable la puesta en marcha de programas poblacionales de detección precoz. Es necesario seguir monitorizando la mortalidad y esperar la publicación de los resultados de los ensayos clínicos randomizados en marcha para poder reevaluar las actuales recomendaciones. 
Tabla 2

Argumentos contra el uso del PSA como prueba de cribado poblacional del cáncer de próstata

1. Altas tasas de prevalencia de cáncer de próstata en necropsias.

2. Una tercera parte de los casos diagnosticados por detección precoz no son curables.

3. A pesar del amplio uso de esta prueba en EEUU, la mortalidad ha permanecido durante muchos años al mismo nivel que la del Reino Unido y otros países europeos, dónde se ha usado mucho menos.

4. No se ha demostrado un claro beneficio del tratamiento.

5. Importantes efectos secundarios de la prostatectomía (impotencia y en menor grado incontinencia).

6. Los costes, tanto del cribado, como del seguimiento y tratamiento (muchos pueden ser innecesarios) son potencialmente muy elevados.

7. En muchos pacientes ancianos el número de años de vida ganados es muy bajo.

8. Todavía no se ha demostrado una reducción de la mortalidad en los ensayos clínicos randomizados.

\section{REFERENCIAS}

1. Jemal A, Thomas A, Murray T, Thun M. Cancer Statistics 2002. Ca Cancer J Clin 2002;52:23-47.

2. Crawford ED. Epidemiology of prostate cancer. Urology 2003;62(6)(Suppl 1):3-12.

3. Boyle P, D'onofrio A, Maissonneuve P et al. Measuring progress against cancer in Europe: has the 15\% decline targeted for 2000 come about?. Annals Oncology 2003;14:1312-1325.

4. Ferlay J, Bray F, Sankila R, Parkin DM. Eucan: Cancer incidence, mortality and prevalence in the European Union 1998, Version 5.0. Iarc Cancerbase No. 4. Lyon, Iarcpress, 1999.

5. Informe sobre cáncer en España, 2003. Área de Epidemiología Ambiental y Cáncer. Centro Nacional de Epidemiologia. Instituto de Salud Carlos III. Madrid [Consulta: 24 De Abril De 2004]. Disponible En: http://193.146.50.130/ cancer/salud-cancer-2003.pdf

6. Haas GP, Sakr WA. Epidemiology of prostate cancer. Ca Cancer J Clin 1997;47:273-287.

7. Steinberg GD, Carter BS, Beaty TH, Childs B, Walsh PC. Family history and the risk of prostate cancer. Prostate. 1990;17(4):337-347.

8. Whittemore AS, Kolonel LN, Wu AH, John EM, Gallagher RP, Howe GR et al. Prostate cancer in relation to diet, physical activity, and body size in blacks, whites, and Asians in the United States and Canada. J Natl Cancer Inst 1995;87(9):652-661.

9. Giovannucci E, Rimm EB, Liu Y, Stampfer MJ, Willett WC. A prospective study of tomato products, lycopene, and prostate cancer risk. J Natl Cancer Inst 2002;94(5):391-398.

10. Clark LC, Dalkin B, Krongrad A, Combs GF Jr, Turnbull BW, Slate EH. Decreased incidence of prostate cancer with selenium supplementation: results of a double-blind cancer prevention trial. Br J Urol 1998;81(5):730-734.

11. Rosenberg DJ, Neugut AI, Ahsan H, Shea S. Diabetes mellitus and the risk of prostate cancer. Cancer Invest 2002;20(2):157-165.

12. INE. Movimiento natural de la población española. Defunciones según la causa de muerte. Vol III. Años 19751991. Madrid: Instituto Nacional de Estadística.

13. IEA. Movimiento natural de la población. Defunciones en Andalucía 1992-1997. Sevilla: Instituto de Estadística de Andalucía.
14. Chin Long Chiang. The life table and its applications. Robert E Krieger Publishing Company Malabar, FL, 1984.

15. Schouten LJ, Straatman H, Kiemeney LA, Verbeek AL. Cancer Incidence: Life table risk versus cumulative risk. J Epidemiol Community Health. 1994;48(6):596-600.

16. Romeder JM, Mcwinnie JR. Potential years of life lost between ages 1 and 70: An indicator of premature mortality for health planning. Int $J$ Epidemiol 1976;6:143-151.

17. López-Abente G, Pollán M, Escolar A, Abraira V. Atlas de mortalidad por cáncer y otras causas en España, 19781992. Fundación Científica de la Asociación Española contra el Cáncer. Madrid, 1996.

18. Bartsch G, Horninger W, Klocker H, Reissigl A, Oberaigner W, Schonitzer D, Severi G, Robertson C, Boyle P. Tyrol prostate cancer screening group. Prostate cancer mortality after introduction of prostate-specific antigen mass screening in the Federal State of Tyrol, Austria. Urology. 2001;58(3):417-424.

19. Consejería de Salud. Plan Integral de Oncológico de Andalucía. Sevilla. Consejería de Salud, 2002.

20. Waterbor JW, Bueschen AW. Prostate cancer screening (United States). Cancer Causes Control. 1995;6(3):267274.

21. Shroder FH, Damhuis RA, Kirkels WJ, de Koning HJ, Kranse R, Nus HG, Blijenberg BG. European Randomized Study Of Screening For Prostate Cancer-The Rotterdam Pilot Studies. Int J Cancer. 1996 Jan 17; 65(2): 145-151.

22. Gómez de Vicente JM, Luján Galán M, Páez Borda A, Romero Cagigal I, Moreno Santurino A, Santos Arrontes D, Berenguer Sánchez A. Detección del cáncer de próstata en el rango de PSA entre 3 y 3,9 ng/ml. Actas Urol Esp 2002;26(4):271-274.

23. Labrie F, Candas B, Dupont A, Cusan L, Gómez JL, Suburu Re, Diamond P, Levesque J, Belanger A. Screening decreases prostate cancer death: first analysis of the 1988 quebec prospective randomized controlled trial. Prostate. 1999;38(2): 83-91.

24. Boer R, Schroder FH. Quebec randomized controlled trial on prostate cancer screening shows no evidence for mortality reduction [letter]. Prostate. 1999;40:130-131.

25. The European Randomized Study of Screening for Prostate Cancer (ERSPC): rationale, structure and preliminary results 1994-2003 Reviews. BJU Int. 2003;92(Suppl 2):1123.

26. Berenguer A, Luján M, Páez A, Santonja C, Pascual T. The Spanish contribution to the European Randomized Study of Screening for Prostate Cancer. BJU Int. 2003Dec;92 Suppl 2:33-38.

27. National Cancer Institute. Division of Cancer Prevention. Prostate, Lung, Colorectal \& Ovarian Cancer Screening Trial (Plco). [Consulta 5 De Abril 2004]. Disponible En: http://www3.cancer.gov/prevention/plco/index.html

28. Harris R, Lohr KN. Screening for Prostate Cancer: An update of the evidence for the U.S. Preventive Services Task Force. Ann Intern Med. 2002 Dec3;137(11):917-929.

29. European code against cancer and scientific justification third version (2003) [Consulta 5 de abril 2004]. Disponible en: http://www.cancercode.org/add_scree01.htm

30. Quinn MJ. Cancer trends in the United States. A view from Europe. J Natl Cancer Inst. 2003 Sep 3;95(17):1258-1261.

31. Barratt A, Mannes P, Irwig L, Trevena L, Craig J, Rychetnik L. Cancer screening. J Epidemiol Community Health. 2002;56(12):899-902.

Dr. M. Ruiz Ramos

Instituto de Estadística de Andalucía

Pabellón de Nueva Zelanda. Leonardo Da Vinci, s/n Isla de la Cartuja - 41071 Sevilla

(Trabajo recibido el 11 mayo de 2004) 